\title{
Settled dust in preschools: characterization and genotoxicity of trace metals and Polycyclic Aromatic Hydrocarbons
}

\author{
REBECCA CASTEL ${ }^{1,2}$, VIRGINIE TASSISTRO ${ }^{1}$, YVES \\ NOACK $^{3}$, MAGALIE CLAEYS-BRUNO ${ }^{1}$, MATHIEU \\ IZARD $^{4}$, FRANCK MAROT ${ }^{5}$, THIERRY ORSIÈRE ${ }^{1}$ AND \\ LAURE MALLERET $^{6}$ \\ ${ }^{1}$ IMBE \\ ${ }^{2}$ Laboratoire de Chimie Environnement (UMR 7376) \\ ${ }^{3}$ CEREGE \\ ${ }^{4}$ ATMOSUD \\ ${ }^{5} \mathrm{ADEME}$ \\ ${ }^{6} \mathrm{LCE}$ \\ Presenting Author: rebecca.castel@imbe.fr
}

Humans are exposed to complex mixtures through their environment. In the last two decades, awareness grew on the matter of the impact of environment on human health and the term of exposome appeared in 2005. However, toxicity effects are generally assessed for single compounds and little is known about toxicity generated by mixtures.

One of the most sensitive populations to chemical exposure are children below 6 years old as they are still growing, and longterm health issues could be increased and appear sooner when exposure occurs in early life stage. An important exposure route for young children is through ingestion as they are still learning to walk, playing on the ground, and bringing their hands and objects to their mouths. Indoor or outdoor settled dusts and soils, with many different compounds orbed onto, could thus be ingested by young children, and raises questions about health impacts. Specifically, trace metals (TM) and polycyclic aromatic hydrocarbons (PAH) are of interest as they are genotoxic and/or carcinogenic and ubiquitous in the environment.

The present study aims to characterize TMs and PAHs in settled dust in pre-schools placed indifferent environmental contexts located in the South of France (Bouches-du-Rhône) and to assess their genotoxicity on gastric cells. Two sampling campaigns (winter/summer) are underway in 5 preschools. To discriminate the input of aerial deposition vs soil to the settled dust, Owen gauges will be installed, and soils will be sampled when possible. Previously analyzed samples served as references to define mixtures with a set of $6 \mathrm{TM}$ and 6 PAHs. The genotoxicity of these mixtures will be studied to correlate dust genotoxicity with the presence of compounds and to investigate possible interactions between these compounds.

This study will allow us to understand the input of soil pollution and aerial deposition of TM and PAH to settled dust and to investigate the possible interactions between compounds in the occurrence of genotoxic hazard, if any. As a result, this research should help better understand hazards of soil or settled dust ingestion, improve the tools for risk assessment of micropollutants mixtures on human health, and better manage contaminated soils. 